\title{
APUNTES PARA UNA HISTORIA DE LAS RELACIONES ENTRE EL JUEGO Y LA RECREACIÓN CON EL TIEMPO LIBRE Y EL TIEMPO DE OCIO EN COLOMBIA A FINALES DEL SIGLO XX \\ NOTES FOR A HISTORY OF THE RELATIONSHIP BETWEEN PLAY AND RECREATION WITH LEISURE TIME AND LEISURE TIME IN COLOMBIA IN THE LATE TWENTIETH CENTURY
}

Claudia Ximena Herrera Beltrán*

\section{Resumen}

\begin{abstract}
Nos ocuparemos en desentrañar de modo somero la emergencia del juego, de la recreación y de la lúdica, de sus diferencias y sus relaciones con el tiempo libre en la segunda mitad del siglo XX en Colombia**. En este sentido, diremos que el juego aparece históricamente como actividad inherente al hombre, pero es hasta el siglo XV cuando se consideró pertinente como actividad humana sin límites etarios; recordemos que la infancia como la conocemos hoy tiene su aparición en el siglo XVI y XVII. A partir de este momento comienzan a ser diferenciados los juegos entre adultos e infantes. Más tarde, las diferencias alcanzarán el sexo y el género en relación con los propósitos a que está destinado cada sujeto; luego, la edad definirá la conveniencia o no de ciertos juegos; lo mismo va a suceder con los juguetes, los que responderán a dichas clasificaciones, fines y propósitos. En este recorrido, mostraremos los discursos en torno al juego libre, su vinculación más con la lúdica y menos con la paideia ${ }^{* * *}$, sus diferencias y relaciones con la recreación en el tiempo de ocio y en el tiempo libre y su uso en cuanto estrategia educativa de niños y jóvenes desde las políticas, la legislación, los proyectos, las teorías y las experiencias en el campo.
\end{abstract}

Palabras claves: paideia, ludus, juego, recreación, ocio, tiempo libre, historia, Colombia.

\section{Abstract}

The purpose of this paper is to unravel the game emergence, recreation and ludic, their differences, and their relationships with free time during the late twentieth century in Colombia. Therefore, we could affirm that the game appears historically as distinctively human activity, but it is up to the fifteenth century when it was considered as an appropriate human activity without age bounds, remember that childhood as we know it today. It first showed up between the sixteenth and the seventieth centuries. From this point, it begins to establish differences between the adult and children's games. Later on, the differences will reach sex and gender related to the purposes for which it is intended each subject; then age will define what games are appropriate according to. The same will happen with toys, those who respond to such categorizations, aims and purposes. On this journey we will show the speeches that revolve around the free game, its relationship with the most ludical and the less "Paideia", their differences and relationships between recreation, leisure and free time and its uses in both children's education strategy and young people from the policies, legislation, projects, theories and experiences in the field.

Keywords: paideia, ludus, game, recreation, leisure, free time, history, Colombia.

Fecha de recepción: 20 de mayo de 2013

Fecha de aceptación: 9 de agosto de 2013

\footnotetext{
* Doctora en Educación, Magíster en Historia de la Educación y la Pedagogía, Licenciada en Educación Física. Profesora de planta de tiempo completo de la Universidad Pedagógica Nacional, Facultad de Educación, Departamento de Posgrado, Maestría en Educación. Correo electrónico: cherrera@pedagogica.edu.co y claudiaximenahb@yahoo.es

** Las primeras motivaciones en torno al tema del juego y su historia en Colombia en la segunda mitad del siglo XX en el ámbito escolar responden a la elaboración de un estado del arte que realicé en diciembre de 2009 para la Universidad Santo Tomás en torno a las ludotecas.

*** La paideia griega enseña a viajar sin método, ya no un viaje en línea recta, más bien una odisea en donde se dibujan caminos fuera de cierto orden.
} 


\section{El contexto}

La infancia aparece con la escuela y la pedagogía. Ambas, en la idea de civilizar. Es el espacio escolar un espacio para pobres, en el que el control y la evangelización ocuparán el lugar central de sus preocupaciones. Entre el siglo XVIII y el siglo XXI, el juego emerge como elemento pedagógico, pasando de ser diversión que requiere vigilancia a elemento didáctico. En y desde la escuela se destierra el juego libre ${ }^{1}$, dándole paso al juego dirigido. Así, los propósitos educativos serán más de orden moralizante e higiénico, un medio para educar armónicamente: física, intelectual y moral. Esta manera de concebir el juego se mantendrá fuertemente hasta mediados del siglo XX, en que otras maneras de utilizarlo desde nuevos saberes y poderes van a hacerse visibles tanto en la escuela como en otros ámbitos sociales en los que otro tipo de educación empieza a tener lugar (Herrera Beltrán y Buitrago, 2011).

En el marco de la escolarización de la infancia ruda entre los siglos XVI y XIX (Varela y Álvarez-Uría, 1997), el juego se supeditó a la infancia, pero con restricciones: perdió su lugar como una actividad sin propósito útil (Duvignaud, 1982), fue vigilada por maestros y adultos $y$, en la medida en que el infante se hacía mayor y maduraba, el juego fue relegado al confín de la infancia perdida, existiendo solamente en el recuerdo. En la escuela -lugar de encierro para ir a civilizarse- se llevó a cabo la regulación de su práctica en espacios y tiempos como el recreo y los descansos, y en las clases fueron usados por los maestros como medio educativo (Barreau y Morne, 1991). La práctica del juego sin autorización y supervisión de los maestros o directivas provocó sanciones y castigos de diversa índole.

Para el caso colombiano, la entrada de las ciencias de la educación a la escuela durante la primera mitad del siglo XX trajo consigo una visibilización de la actividad lúdica -como señalábamos arriba-; el juego se hizo recurso educativo especialmente desde saberes como la psicología, que le concedió un lugar fundamental en el desarrollo del niño. La psicología, ocupada del estudio del infante, de las maneras como aprendía, de su desarrollo físico y la relación con su desarrollo intelectual y mental, en suma, un saber fundamentalmente etario, preocupado por los fenómenos educativos, impulsó al maestro hacia un oficio altamente experimental, cuya aplicación se efectuaba sobre el infante y desde una

1 Se entenderá juego libre como una categoría metodológica usada para situar el juego de los niños en el que no interviene de ningún modo el adulto. metodología que usaba el juego, entre otros medios, para alcanzar sus propósitos. Lo anterior, aunado a la construcción de los estados nacionales, contribuyó a educar para el progreso ${ }^{2}$. De allí que fuera posible modernizar al país a través de la escuela (Sáenz, Saldarriaga y Ospina, 1997), desde las ciencias de la educación: de las ciencias experimentales y especialmente desde la psicología.

Es de señalar que otra cosa fue la que ocurrió en la segunda mitad del siglo XX, allí, el desarrollo desde una perspectiva económica reconfiguró los fines, los medios sociales y educativos que hoy nos constituyen (Escobar, 2007). Lo que no resulta una continuidad, al contrario, será una ruptura en la cual la educación pasará a ser más que nada un asunto técnico y una inversión social importante -educación y desarrollo constituirán la dupla por excelencia del reordenamiento del mundo, que a su vez conducirá a superar las dificultades del subdesarrollo-, orientado su funcionamiento principalmente desde los principios de eficacia y rentabilidad. Se suman a estos elementos el carácter mundial que adquiere lo educativo frente a la consideración nacional de finales del siglo XIX y comienzos del siglo XX (Martínez Boom, Noguera Ramirez y Castro, 2003).

Para los países nombrados como del tercer mundo, en la década de los cincuenta se inició una dinámica pro desarrollo, por cuanto dichas naciones fueron consideradas subdesarrolladas. Así, la educación en relación con el desarrollo se vio envuelta en compromisos económicos de corte multinacional, condicionados a estudios que ahondaban en las crisis educativas que atravesaban estos países y que podían solucionarse solo si se utilizaba la ciencia y la tecnología. Dichos presupuestos posibilitaron la entrada de la teoría de sistemas y, más tarde, de la tecnología educativa al campo educativo y pedagógico, lo que hizo posible la transferencia de la tecnología a la educación y la enseñanza. Así fue como la planificación económica a partir de sus principios y métodos, llegó y produjo lo que se denominó el planeamiento integral de la educación reemplazada en la década de los setenta por la tecnología educativa, más tarde nombrada como tecnología instrumental. Si bien estas teorías, métodos, procedimientos y principios estuvieron al margen de las teorías, ellas en sí mismas

2 No podemos olvidar la relación que el progreso tendrá históricamente con las formas modernas de gobierno y desde posturas teóricas diversas. Diremos que la relación estado nación o estado nacional, proyecto moderno de los siglos XVIII y XIX estará fuertemente vinculado con el progreso en el orden de la ciencia y la tecnología, idea que encontramos en pensadores franceses como Turgot, SaintSimon y Auguste Comte, este último y sus seguidores positivistas relacionarán el progreso humano con la ciencia y la industria. 
van a constituir lo que Martínez Boom, Noguera y Castro denominaran Campo del Currículo (2003) -categoría metodológica- que fue, sobre todo, una teoría sobre la instrucción y cuyo fin primordial sería la obtención de un aprendizaje efectivo.

De otro lado, la ampliación antes que la profundización de los estudios sobre el niño y el joven -emerge el joven como objeto de estudio, e intervención- en la segunda mitad del siglo XX hasta la actualidad muestra amplios desarrollos escolares y sociales donde el juego o la actividad lúdica ha adquirido nuevas dimensiones. Los fines educativos privilegiaron más que la formación individual encaminada a una educación donde el trabajo colectivo era esencial, hacia una formación del hombre como capital humano inscrito en las lógicas del mercado, donde rendir, producir de manera eficaz y con calidad tomaron fuerza. En este contexto económico particular, bajo la tecnología educativa y la curricularización de la educación (Martínez Boom, Noguera Ramirez y Castro, 2003), aparece la recreación como práctica que irá configurando un saber que para ese momento resulta urgente y necesario. En este proceso, se vuelve natural recrear y recrearse -para ocupar el tiempo de ocio, el tiempo libre de los hombres, de los niños, de los jóvenes ${ }^{3}$ - así, la recreación va a configurarse en un dispositivo de control de la población puesto a funcionar mediante estrategias diversas, como fueron "el deporte para todos", "los aeróbicos" y "la ciclovía", entre otros. Más adelante, la lúdica discurso, que expresa antes que nada un modo divertido de aprender que no necesariamente significa juego, se pone a funcionar en todas las acciones humanas educativas no únicamente pedagógicas o referidas al entorno escolar. Con ella, se justifican nuevas estrategias educativas como las ludotecas, cuya aparición se debe además a una necesidad de proveer mecanismos que acerquen, estimulen y desarrollen a los niños en condiciones económicas, sociales y culturales desfavorables mediante actividades lúdicas realizadas en espacios similares a

3 Diferenciamos tiempo de ocio de tiempo libre a partir de la idea del tiempo y su aprovechamiento: el tiempo ocio será un espacio en que el individuo se ocupe de algo que le interesa a él y solo a él, al margen de si su accionar le representa un beneficio, realizado en cualquier momento solo bajo su deseo de realización, el tiempo libre lo entendemos asociado al trabajo, es un tiempo que hay entre jornadas de trabajo en el mismo día o de un día para otro, tiempo en que se realizan actividades urgentes y cotidianas que el trabajo mismo no permite realizar, pues no deja tiempo para ellas. También van a convertirse en los tiempos privilegiados para desarrollar actividades útiles al trabajo que se realiza, incluso actividades físicas que se leerán a partir de la década de los ochenta como necesarias al rendimiento y la producción en el trabajo por cuanto aportan un mejor estado físico (Elías y Dunning, 1992). las bibliotecas ${ }^{4}$, así llega el juego hasta nuestros días, naturalizado desde la escuela y en relación estrecha con el niño, justificado especialmente desde la psicología como fundamental para el desarrollo de la infancia y su incorporación al mundo adulto; el juego se hará visible e importante en el ámbito social en el que es ahora necesario educar para producir.

El hombre, como hemos dicho, se tornará en capital, capital humano, para lo cual es necesario procurarle cuidado y bienestar; de ello dependerá la economía, el desarrollo de las naciones y el futuro del mundo capitalista, y a ello contribuirá la escuela: instruir y civilizar al hombre del siglo XIX, a la educación del hombre para una sociedad que progresa, pasando en las décadas de los ochenta y noventa del siglo XX a una educación del hombre como capital humano, en el marco del desarrollo en una ciudad educadora -ya no será solamente la escuela la que educa, entraran otras instituciones, espacios y tecnologías- y mediante una pedagogía ciudadana (Saldarriaga, 2003); donde la calidad y la eficiencia serán los raseros que muestren el nivel de desarrollo alcanzado por un país o una región.

Pero el juego no ha estado solo, en la primera mitad del siglo XX ocuparon también un lugar importante la educación física y el deporte. La educación física apareció como un saber escolar a finales del siglo XIX en Colombia, consolidándose en la primera mitad del siglo XX una invención de los pedagogos de la Escuela Nueva en la idea de configurar un sujeto nuevo para una nación que se perfilaba moderna (Herrera Beltrán C. X., 1999). El deporte, entre tanto, se convirtió para nuestra cultura en una herramienta importantísima a la hora de educar y conducir a las poblaciones juveniles generalmente pobres mediante el encauzamiento de sus impulsos y de sus instintos, a la vez que recurso eficaz para mantener la salud, cuidar el medio ambiente $\mathrm{y}$ fortalecer los lazos nacionales e internacionales.

Estos saberes fueron validados a comienzos del siglo $\mathrm{XX}$ por pedagogos y políticos y viabilizados en los espacios escolares y sociales en la segunda mitad del siglo XX. Dan cuenta de ello algunas políticas sociales, como la creación de Coldeportes Nacional (Ministerio de Educación Nacional, 1968), que primero se llamó Instituto Colombiano del Deporte y la Juventud en 1968, desde donde se impulsaron campañas como "Deporte para todos", iniciada en el mundo en 1990

4 En dichos lugares se esperaba situar diversos objetos, juegos, actividades que contribuyeran a mejorar las condiciones motrices y cognitivas de la infancia vulnerable. 
como una clara intención de estimular mediante un programa de gran magnitud la participación, la sana competencia y los valores de la salud y el deporte de un grupo de países europeos, expandida a América y otras regiones. Tuvieron lugar en América Latina conferencias panamericanas del deporte para todos, en Buenos Aires (I) en Guatemala (II) en Bogotá (III) en 1989 y en Uruguay, entre otros; dichas reuniones hicieron posible la firma del convenio internacional que aprobaba los estatutos para Colombia y los países participantes del creado Consejo Iberoamericano del Deporte (Consejo Iberoamericano del Deporte, 1994), así como encuentros, conferencias, simposios y congresos cuyo eje central era la educación integral y necesaria a los fines desarrollistas de nuestro continente. El posicionamiento del deporte durante la segunda mitad del siglo XX, como parte importante de las prácticas corporales escolares y sociales, fue relegando al juego libre a una actividad menor -no así como medio didáctico de los saberes escolares y actividad imprescindible en la educación infantil preescolar y primaria desde el saber psicológico-. Ya en la década de los setenta, el juego comienza a cobrar valor fuera de la institución escolar, contribuyendo a la emergencia del saber denominado recreación que se institucionalizara.

Así, cada uno de ellos, la recreación, el deporte (Serrano Sánchez, 1992) y la educación física durante la segunda mitad del siglo XX se constituirán en actividades sociales erigiéndose en derechos ${ }^{5}$ del ciudadano (UNESCO, 1978) desde las políticas educativas internacionales (UNESCO, 1976) y también en servicios sociales desde la legislación colombiana (Congreso de la República de Colombia, 2004) ${ }^{6}$. Con esta transformación nominati-

5 Artículo 1. Que la práctica de la educación física y el deporte es un derecho fundamental para todos 1.1. Todo ser humano tiene un derecho fundamental de acceso a la educación física y el deporte, que son esenciales para el pleno desarrollo de su personalidad. La libertad de desarrollar facultades físicas, intelectuales y morales a través de la educación física y el deporte debe estar garantizada tanto dentro del sistema educativo y en otros aspectos de la vida social. 6 Artículo 44. Son derechos fundamentales de los niños: la vida, la integridad física, la salud y la seguridad social, la alimentación equilibrada, su nombre y nacionalidad, tener una familia y no ser separados de ella, el cuidado y amor, la educación y la cultura, la recreación y la libre expresión de su opinión. ARTICULO 52. (Modificado. Acto Legislativo 2 de 2000 Artículo 1)...Se reconoce el derecho de todas las personas a la recreación, a la práctica del deporte y al aprovechamiento del tiempo libre. El Estado fomentará estas actividades e inspeccionará, vigilará y controlará las organizaciones deportivas y recreativas cuya estructura y propiedad deberá ser democrática. Texto Derogado por el Acto Legislativo 2 de 2002, Art. 1 ARTICULO 64. ARTICULO 67. La educación es un derecho de la persona y un servicio público que tiene una función social; con ella se busca el acceso al conocimiento, a la ciencia, a la técnica, y a los demás bienes y valores de la cultura. La educación formará al colombiano en el respeto a los derechos humanos, a la paz y a la democracia; y en va, se adelantaron una serie de discusiones alrededor de los términos: ocio, tiempo libre, creatividad, lúdica y, por supuesto, recreación.

Si bien la historia de la recreación está estrechamente vinculada con la institucionalización del tiempo libre, en la misma medida lo está respecto al trabajo, a la constitución de la democracia, a la paz y, en general, a la formación de las identidades nacionales. Caracterizar y precisar sus límites como disciplina (Mesa Cobo, 1999) supone definir, situar y contextualizar histórica, social y epistemológicamente dichos conceptos y mostrar sus relaciones. Tarea de la que no nos ocuparemos en esta elaboración más que de manera somera. Diremos solamente que de la paideia se pasó al ludus (Caillois, 1986), del ocio en Grecia y Roma, se comenzó a hablar del tiempo libre, categoría referida al tiempo del no trabajo en el siglo XVII con la emergencia del gobierno liberal, donde el capital será central para pensar las relaciones y las nuevas subjetividades; ya en el siglo XX, la recreación emerge justificada desde discursos diversos que apuntan a su institucionalización y normalización especialmente para la infancia y la juventud, como recurso importante a la hora de educar para la producción. Ella ha visto aparecer un corpus teórico, y un corpus práctico amplísimo, especialmente en la segunda mitad del siglo $\mathrm{XX}$, que se ha tornado connatural a la sociedad; como categoría política y desde muchos saberes y poderes se muestran sus beneficios y se le implementa bajo la justificación de una necesidad. Así, la recreación se vive en la colectividad, de manera masiva impulsada desde los medios de comunicación, la publicidad y desde instituciones y políticas multinacionales que siguen muchos de los principios que fundamentan el juego. La recreación tendrá como propósitos la organización y el control social mediante actividades que dan salida a las pulsiones de manera regulada y beneficiosa.

La normalización de las actividades y las prácticas humanas, así como el aprovechamiento del tiempo en actividades útiles y productivas, será lo que rija las dinámicas sociales, las relaciones entre los sujetos, los modales, las formas de hablar y de divertirse. El tiempo libre es entonces un concepto estrechamente producido desde el trabajo y como oposición al mismo, es decir, aquel que trabaja tiene derecho a descansar y en ese tiempo, tiempo libre, se recreará, ya no desde su libre

la práctica del trabajo y la recreación, para el mejoramiento cultural, científico, tecnológico y para la protección del ambiente. CONSTITUCIÓN POLÍTICA DE COLOMBIA Actualizada con todos los Actos Legislativos expedidos hasta el 2004. Recuperado el día 19 de junio del 2013. Recuperado de http://www.secretariasenado.gov.co/senado/basedoc/cp/acto_legislativo_02_2004.html 
albedrío (Sáenz, Saldarriaga y Ospina, 1997),7 pues se le ofrecerán actividades recreativas por doquier. Uno de esos espacios distintos y novedosos fueron las empresas que a partir de la década de los sesenta contaron con las llamadas cajas de compensación familiar, creadas en los años cincuenta simultáneamente con la implementación del sistema de subsidio familiar encargado del bienestar de los empleados mediante actividades de bienestar social, nombradas como recreación a las empresas afiliadas. Estas estrategias y discursos emergieron desde el Ministerio de Trabajo y Bienestar Social ${ }^{8}$ y desde los colegios y universidades, como de los barrios y las alcaldías. Esas actividades para el tiempo libre tuvieron una gran oferta, igual fueron en aumento los sitios que vendían los objetos de consumo para recrearse: juguetes, juegos, ropa e implementos deportivos, objetos muy diversos, así como los profesionales en recreación especializados en el mejor aprovechamiento del tiempo libre. La recreación se constituyó para la segunda mitad del siglo $\mathrm{XX}$ en un derecho, un servicio y un bien de consumo.

Este aumento del tiempo libre en las sociedades capitalistas responde a una necesidad estructural del sistema: descansar para seguir produciendo y disponer de tiempo para el consumo (Mesa Cobo, 1999). Un descanso que debe ser, a todas luces, reparador, que sirva de acondicionamiento físico para el trabajo, que represente bienestar, salud, beneficios. Un ejemplo de ello pero que estuvo dirigido a la infancia, objeto también de atención en tanto capital humano- fueron las ludotecas que nacen para la infancia necesitada y desprotegida, extendiéndose posteriormente a grupos humanos o comunidades desplazadas y en riesgo; familias, adultos mayores y jóvenes como un modo de recreación.

7 Estos autores muestran en su libro Mirar la infancia cómo en la primera mitad del siglo XX "la desconfianza en el pueblo" se constituyó en una rejilla por la que la modernización entró al país. Dicho de otro modo, se instaló en los discursos una cierta incapacidad del sujeto y del pueblo de decidir su propio destino, situación que justificaba, entre otras cosas, direccionar desde los poderes políticos y sociales el uso del tiempo libre, es decir, en qué recrearse.

8 Las cajas de compensación familiar en Colombia tienen su origen hacia 1954, cuando la Asociación Nacional de Industriales (Andi) sugirió al gobierno establecer el subsidio familiar en todo el país para atender las necesidades de los hogares de los obreros, en proporción al número de hijos. Si bien la función básica de las cajas en sus inicios se limitaba, según lo ordenaban las normas vigentes en ese entonces, a recaudar los aportes patronales para repartirlos mensualmente en cheques a los trabajadores de las empresas afiliadas, es en 1962 cuando el Ministerio de Trabajo y Seguridad Social, a través del decreto 3151 del mismo año, abrió las puertas a los servicios sociales de las cajas de compensación familiar; fue así como nacieron los servicios de salud, mercadeo, educación, vivienda, recreación y crédito de fomento para industrias familiares. Recuperado de http://www.colsubsidio.com/es_caja/index.html\#d
Pero, ¿cómo se definen el juego, la recreación, el ocio, la lúdica? ¿Cuáles son sus propósitos? ¿En qué contextos aparecen? ¿Quiénes las ponen a funcionar? ¿Quiénes emiten discursos sobre la conveniencia de estas actividades? ¿Cómo se diferencian? ¿Cómo se relacionan entre si dichas categorías? ¿Quiénes practican estas actividades?

\section{Historia y genealogía de los conceptos}

Si como lo afirma Caillois (1986), al juego libre y al juego dirigido se les puede situar entre dos polos opuestos nombrados como paideia y ludus, en uno de cuyos extremos reina un principio común de diversión, de turbulencia, de libre improvisación y de despreocupada plenitud, a través de la cual se manifiesta cierta fantasía desbocada que podemos designar mediante el nombre de paideia; mientras que en el otro extremo reina esa exuberancia traviesa y espontánea absorbida y disciplinada, aparece una necesidad siempre creciente de plegarla a convencionalismos arbitrarios, imperativos y molestos. A este segundo componente lo llamó ludus (Caillois, 1986). Dicho de otro modo, si aceptamos que el ludus es la pedagogización de la paideia -del goce al placer permitido-, diremos que del juego libre sin propósito más allá del goce, se pasó al juego dirigido en tiempos asignados especialmente: el del recreo, la clase de educación física -antes de calistenia o de gimnasia, los paseos en domingo y las vacaciones. Esa manera de jugar tuvo como propósito fundamental el control de los instintos que traía a los niños terribles consecuencias, desde la mirada vigilante de maestros y padres quienes buscaban a toda costa "fertilizar y vacunar el alma infantil contra su virulencia" (Caillois, 1986).

Otro tanto le sucedió al mundo del juguete ${ }^{9}$-inmenso y fascinante-, que terminó pensado para el niño por el adulto. Ya describía Philippe Aries cómo en el Antiguo Régimen los juguetes fueron elaborados por los adultos para los adultos y no para los niños, y que poco a poco, dada la importancia que tomó la infancia -los sentimientos que surgieron alrededor de ella-, en una ciudad que se construía socialmente para una familia cada vez más reducida, estos juguetes fueron pasando a las manos de los niños infantilizándolos, olvidados ya por los mayores como objetos de gozo y diversión (1987). Si el juguete fue entonces un dispositivo ahora de la infancia (Deleuze, 1989), el juego pasó a ser una

9 Puede entenderse el juguete como un dispositivo de subjetividad en el que tiene lugar importante el poder. Así, el juguete contribuye en cuanto dispositivo-máquina estratégica "para hacer ver y para hacer hablar" a configurar al tipo de infante que se desea. 
tecnología disciplinaria ${ }^{10}$ usada en la escuela y posteriormente en otros espacios sociales (Foucault, 1990) en las décadas de los setenta y ochenta, como veremos más adelante.

Algunos saberes que entraron a la escuela en esta época en Colombia, como la medicina, y otros que se produjeron en su interior, como la higiene, la educación física y la urbanidad, entre otros, reglaron, prohibieron y autorizaron los juegos para la infancia desde principios morales e higiénicos. A finales del siglo XIX y comienzos del siglo XX, se consideraba que cada institución escolar debía tener un patio o lugar de juego libre donde los alumnos pudieran gozar de su recreación sin interrupciones externas y sin influencias que el maestro no pudiera dominar. También los juegos dirigidos servían al maestro para enseñar a los niños la forma de relacionarse con los otros; los modales, las actitudes, los comportamientos anormales y el deber ser, permitiendo al maestro reconocer las habilidades manuales infantiles para el trabajo posterior, (Caillois, 1986) ${ }^{11}$ sirvieron como fundamentos iniciales de actividades laborales y ayudaron a clasificar a los niños según enfermedades o desarreglos mentales, impulsando, según la edad y la condición de salud, diversos deportes ${ }^{12}$.

El juego fue en sus comienzos un medio fundamental de la educación física -saber escolar de aparición reciente y cuya impartición por profesionales solo fue posible a partir de 1936. Entre 1870 y 1936 estuvo a cargo de maestros de escuela, policías y encargados de la disciplina, etc.-. Las corrientes de la educación física escolar que llegaron a Colombia promovían ampliamente el juego, entre ellas podemos destacar la gimnasia sueca

10 "Las tecnologías de uno mismo son aquellas técnicas que permiten a los individuos efectuar un cierto número de operaciones en sus propios cuerpos, en sus almas, en sus pensamientos, en sus conductas y ello de un modo tal que los transforme a sí mismos, que los modifique, con el fin de alcanzar un cierto estado de perfección, o de felicidad, o de pureza, o de poder sobrenatural, etc.".Debemos comprender que existen cuatro tipos principales de estas tecnologías y que cada una de ellas representa una matriz de la razón práctica: tecnologías de la producción, tecnologías de sistemas de signos, tecnologías de poder y tecnologías del yo. Estos cuatro tipos de tecnologías casi nunca funcionan de modo separado, aunque cada una de ellas este asociada con algún tipo de particular de dominación. Cada una implica ciertas formas de aprendizaje y de modificación de los individuos, no solo en el sentido más evidente de adquisición de ciertas habilidades, sino también en el sentido de ciertas actitudes". 11 "Aprenden a hacer uso de los juegos de sociedad como juguetes comunes, y por medio de ellos pueden dar expresión a sus ideas colectivas sobre asuntos de interés social".

12 En los periódicos y revistas dedicadas a la educación en Colombia circularon gran cantidad de artículos referidos a los juegos de los niños, su pertinencia y su importancia. Del Real y Mijares, 1906, p. 90.
-Ling ${ }^{13}$ y Janh ${ }^{14}$ fueron sus promotores desde Suecia-, en la que el juego fue considerado como un medio de alto valor educativo.

Para todos estos saberes, ciencias y disciplinas, fue claro que el juego procuraba distracción y alegría, resultando ser un excitante poderoso. De allí que se considerara al juego un excelente médico del cuerpo, debido al placer que le procuraba al infante al jugar. Se afirmaba que nada podría reemplazar a los juegos dirigidos al aire libre, con los cuales se exaltaban todas las energías vitales, se desarrollaban los órganos, se armonizaban todas sus funciones, se educaba moral e intelectualmente; los juegos superaban ampliamente los alcances de la gimnasia racional y la de los medios ortopédicos más científicos y de avanzada (Herrera Beltrán y Buitrago, 2011).

Así llega el juego hasta nuestros días, naturalizado desde la escuela y en relación estrecha con el niño, justificado especialmente desde la psicología, fundamental para el desarrollo de la infancia y su incorporación al mundo adulto; de este modo, el juego se hará visible e importante en el ámbito social, en el que es ahora necesario educar para producir en cuanto capital de un mundo en desarrollo. Así, la escuela en las postrimerías del siglo XX pasará de instruir y civilizar al hombre a educarlo para una sociedad que progresa; ya en las décadas de los ochenta y noventa del siglo XX se pretende educar al hombre como capital humano para el desarrollo y la ciudadanía, desde una ciudad educadora y mediante una pedagogía ciudadana (Saldarriaga, 2003), donde la calidad y la eficiencia son los raseros a partir de los cuales se evidencia el desarrollo alcanzado por el país.

Históricamente, otras prácticas como las actividades deportivas fueron reemplazando a las actividades festivas, sin límite y fuertemente tánicas, por una diversión más civilizada, previsible, reglada, localizable y jerarquizada. Esta nueva dinámica de la competición llevada hacia el trabajo implicó la noción de rendimiento, la productividad y la eficiencia; la relación medios-fines,

13 Educador, poeta y maestro de armas, nació en 1776 en Lejunga Suiza; en 1813 fundó su Instituto Central de Gimnasia, que dirigió hasta 1836; dejó a su muerte los originales del escrito Los principios generales de la gimnasia; se le considera el padre de la gimnasia sueca: la gimnasia debe estar en armonía con las leyes del organismo humano.

14 Filósofo y educador prusiano que en su obra La nacionalidad alemana, escrita en 1810, enuncia desde ya la importancia de los ejercicios físicos para la agilidad y fuerza del muchacho alemán; en 1816, escribe la obra titulada La gimnasia alemana. Su método mostraba una fuerte tendencia militar. 
la organización y distribución de actividades individuales y colectivas, la puesta en acción de la racionalidad instrumental, apoyada en el modelo mecanicista donde la actividad/productividad de cada persona, ubicada en el lugar que le corresponde, es uniforme y medible y puede ser supervisada, así como sustituida por otra que realice la misma función de un modo más productivo. De allí el apelativo de capital humano.

Así, el deporte, saber, disciplina o ciencia reciente como se le sitúa en el contexto de las ciencias sociales y humanas-, es nombrado como espectáculo y fenómeno social que se configurará con una didáctica propia (Barreau y Morne, 1991). En los círculos académicos educativos, la consideración del deporte como hecho social le permitirá prescindir de la educación física para existir, ocupando, en cambio, espacios urbanos y tiempos sociales fuera del espacio escolar, lo que lo hará más educativo que pedagógico. El deporte se convertirá para nuestra cultura en una herramienta importantísima a la hora de educar y conducir a las poblaciones juveniles generalmente pobres mediante el encauzamiento de sus impulsos y de sus instintos. También es un recurso eficaz para mantener la salud, cuidar el medio ambiente y fortalecer los lazos nacionales e internacionales; discursos validados a comienzos del siglo XX por pedagogos y políticos y viabilizados en los espacios escolares y sociales en la segunda mitad del siglo XX.

Dan cuenta de ello políticas sociales como la creación de Coldeportes Nacional (Ministerio de Educación Nacional, 1968) ${ }^{15}$, llamado primero Instituto Colombiano del Deporte y la Juventud en 1968, desde donde se impulsaron campañas como "Deporte para todos", cuyo inicio en el mundo se produjo en 1990 como una clara apuesta de un grupo de países europeos, que luego se extendió a América y otras regiones. Tuvieron lugar en América Latina conferencias panamericanas del deporte para todos, en Buenos Aires, en Guatemala, en Bogotá en 1989 y en Uruguay, entre otros; dicha reunión dio paso a la firma del convenio internacional que aprobaba para Colombia y los países participantes los Estatutos del Consejo Iberoamericano del Deporte (Consejo Iberoamericano del Deporte, 1994), así como encuentros, conferencias, simposios y congresos, cuyos ejes centrales fueron el deporte, la educación física y la recreación,

15 Como aparece en el Artículo 1. Créase el Consejo Nacional de la Juventud y el Deporte como órgano consultivo del gobierno nacional en todo lo relacionado con la política de bienestar y recreación de la juventud y desarrollo de la educación física y el deporte en toda la nación. fundamentales en la educación integral y necesaria a los fines desarrollistas de nuestro continente.

En dichos estatutos se afirmaba lo siguiente: el deporte es valorado hoy, en todo el mundo, como una actividad con un importante y significativo papel educativo y cultural. La iniciación y la práctica deportivas en el medio escolar o fuera de él, ya sea para los niños y jóvenes como para los adultos, constituyen elementos educativos clave para el desarrollo humano, tanto desde la perspectiva individual como colectiva. Manifestaban que el deporte se ha convertido en el fenómeno cultural de masas más importante de este siglo. Practicado en todos los países del mundo por cientos de millones de personas y seguido como espectáculo por miles de millones a través de los medios de comunicación. Asimismo, indicaron que no existe un fenómeno cultural equivalente que haya tenido un desarrollo tan significativo en el presente siglo traspasando las fronteras de los países, de los continentes, de las culturas, de las razas, de las clases sociales o de las ideas políticas. Expresaron que la práctica deportiva se constituye como elemento clave en la salud tanto del individuo como de la sociedad. Por otra parte, se señala que la actividad deportiva coadyuva a respetar los recursos naturales del planeta y a su correcta utilización. Reafirmaron que la práctica sistemática del deporte fomenta y transmite un conjunto de valores positivos de gran importancia para la sociedad como: el esfuerzo, el autocontrol, la constancia y la tenacidad, la solidaridad y el trabajo en equipo. Esta escuela de la vida ayuda a la integración comunitaria y al desarrollo social, constituye un elemento de prevención de conductas antisociales, eleva la calidad de vida y su práctica, a través de una amplia gama de organizaciones deportivas, representa una verdadera escuela de democracia.

Otros saberes como la gimnasia aportaron sus investigaciones a hacer del hombre-máquina, lo más eficiente posible. Es el caso de los aeróbicos, método de acondicionamiento físico diseñado por Kenneth Cooper ${ }^{16}$, difundido a todo Occidente como la mejor forma de lograr y mantener la salud física del hombre. Este mo-

16 Kenneth H. Cooper (4 de marzo de 1931, Oklahoma). Doctor en medicina y coronel de la Fuerza Aérea de los Estados Unidos. Cooper es el autor del libro Aerobics (1968), en el que diseñó un método para mejorar el sistema cardiovascular. En 1970 publicó una nueva versión con gran aceptación en el mercado, The New Aerobics. Durante su carrera militar, Cooper diseñó el test de Cooper como una forma sencilla y rápida de evaluar la condición física de un elevado número de personas. Cooper es el fundador del Centro de Aeróbicos Cooper en Dallas, Texas, y del Instituto Cooper, abierto en 1970 como una organización educacional y de investigación sin ánimo de lucro. Recuperado de http://es.wikipedia.org/wiki/Kenneth_H._Cooper 
delo entró con fuerza y se hizo práctica recreativa útil y eficiente a la hora de trabajar, garantizando una buena condición de salud con menos gastos en tratamientos, medicinas e incapacidades laborales. Acogida por las empresas de manera entusiasta, constituyó parte de las actividades deportivas recreativas y lúdicas que hoy de manera más especializada se ofrecen en el mercado como necesarias, con nombres nuevos pero con propósitos similares: la gimnasia aeróbica, la danza aeróbica, los aeróbicos, Danzika, Pilates, Bodypump, Bodycombat, rumba, etc.

En Colombia, la campaña "deporte para todos" llevó a la calle la actividad recreativa en la idea de brindar a la población en general actividades que contribuyeran al cuidado de la salud mediante acondicionamiento físico en grupos. Las actividades deportivas y recreativas proveían básicamente de elementos aeróbicos, ejemplo de ello, fueron las sesiones masivas de gimnasia aeróbica los días festivos en plazas y parques ${ }^{17}$, así como la implementación de las ciclovías, que aparecieron entre 1974 y 1976 y que siguen hoy ofreciendo un espacio para el esparcimiento y la recreación donde la práctica de los deportes de ruedas como caminar y correr son su centro. Ambas resultaban estratégicas a la hora de cuidar la salud de la población. De ello devino la readecuación y construcción de parques y zonas deportivas en toda la ciudad.

El posicionamiento del deporte dentro de las prácticas corporales escolares y sociales fue relegando al juego a una actividad menor -no así como medio didáctico de los saberes escolares y actividad imprescindible en la educación infantil preescolar y primaria desde el saber psicológico ahora pedagogizado-; aunque simultáneamente comienza a cobrar valor fuera de la escuela, lo que contribuyó a la emergencia del saber denominado recreación que comienza a ser institucionalizado. El deporte, en tanto y por algún tiempo, ocupará los primeros lugares en los fines educativos del saber de la educación física (UNESCO, 1978) ${ }^{18}$; después se independizará de dicho saber escolar -aunque continúe siendo un

17 Dichas actividades son mencionadas por Óscar Azuero en el marco de la Conferencia Panamericana del Deporte para Todos, realizada en Bogotá en 1989 en las instalaciones del Club Militar. Nota en el periódico El Tiempo el 18 de abril de 1989.

18 En la carta internacional se educación física y deporte de la Unesco de 1978 se afirma en el artículo 1 que la práctica de la educación física y el deporte son un derecho fundamental para todos, que todo ser humano tiene un derecho fundamental de acceso a la educación física y el deporte, que son esenciales para el pleno desarrollo de su personalidad. La libertad de desarrollar facultades físicas, intelectuales y morales a través de la educación física y el deporte debe estar garantizada tanto dentro del sistema educativo como en otros aspectos de la vida social. medio educativo importante ${ }^{19}$-. Así, de modo paralelo, la recreación, el deporte y la educación física avanzarán como actividades sociales, constituyéndose en derechos del ciudadano desde políticas educativas internacionales (UNESCO, 1976) ${ }^{20}$ y a partir también de la legislación colombiana (Congreso de la República de Colombia, $2004)^{21}$. No podemos olvidar que el deporte le aportó a la educación física como saber escolar en la primera mitad del siglo XX su representatividad al hacer parte de dicha práctica escolar hasta entrada la década del 80 , donde la crisis de estos saberes produjo grandes discusiones como rupturas, de las cuales podemos nombrar la separación entre los estudios de educación física, deporte y recreación en la Universidad Pedagógica Nacional en Bogotá, separación que hoy sigue requiriendo análisis de lo acontecido entre ese momento de discusión, su separación curricular en el 2000 y lo vivido hasta hoy como programas independientes.

Con esta transformación nominativa vienen aparejadas profundas modificaciones a su comprensión conceptual: epistemológica y metodológica, produciéndose

19 Es importante señalar que la educación física escolar fue retirada de la escuela primaria colombiana en el ámbito público, es decir, no fue posible volver a contar con maestros especializados en este saber para la escuela pública primaria, decisión legislativa que viene haciendo crisis en la educación infantil desde la década de los noventa.

20 Se recomienda crear, a través de los organismos pertinentes, las condiciones que permitan el trabajo y el ocio, cada uno a su manera, ofrecer oportunidades para la creación cultural a todos y cada uno y establecer condiciones de trabajo y horas de ocio y la organización operativa de las instituciones culturales que permitan el mayor número posible de personas a tener acceso a la cultura y participar en la vida cultural.

21 En algunos de los artículos de la Constitución Política de Colombia de 1994 reformados en 2004 se recogen algunos de los derechos que se instituyen especialmente en torno a la recreación, el tiempo libre, el juego y el deporte: Artículo 44. Son derechos fundamentales de los niños: la vida, la integridad física, la salud y la seguridad social, la alimentación equilibrada, su nombre y nacionalidad, tener una familia y no ser separados de ella, el cuidado y amor, la educación y la cultura, la recreación y la libre expresión de su opinión. Artículo 52. (Modificado. Acto Legislativo 2 de 2000 Artículo 1) El ejercicio del deporte, sus manifestaciones recreativas, competitivas y autóctonas tienen como función la formación integral de las personas, preservar y desarrollar una mejor salud en el ser humano. El deporte y la recreación, forman parte de la educación y constituyen gasto público social. Se reconoce el derecho de todas las personas a la recreación, a la práctica del deporte y al aprovechamiento del tiempo libre... Artículo 52. (Texto Aprobado por la Asamblea Nacional Constituyente de 1991) Se reconoce el derecho de todas las personas a la recreación, a la práctica del deporte y al aprovechamiento del tiempo libre. El Estado fomentará estas actividades e inspeccionará las organizaciones deportivas, cuya estructura y propiedad deberán ser democráticas. Texto Derogado por el Acto Legislativo 2 de 2002, Art. 1 Artículo 64. Es deber del Estado promover el acceso progresivo a...los servicios de educación, salud, vivienda, seguridad social, recreación, crédito, comunicaciones, comercialización de los productos, asistencia técnica y empresarial, con el fin de mejorar el ingreso y calidad de vida de los campesinos. Artículo 67. La educación formará al colombiano en el respeto a los derechos humanos, a la paz y a la democracia; y en la práctica del trabajo y la recreación, para el mejoramiento cultural, científico, tecnológico y para la protección del ambiente. 
una serie de discusiones alrededor de los términos: ocio, tiempo libre, creatividad, lúdica y por supuesto recreación.

Respecto de la recreación, saber de aparición reciente -aunque ya se le mencionada en un libro de economía por la década de 1880-, es en la segunda mitad del siglo XX cuando se le sitúa entre las disciplinas sociales y humanas desde múltiples discursos venidos de lugares distintos. Si bien la historia de la recreación está estrechamente vinculada con la institucionalización del tiempo libre -distinto del ocio-, en la misma medida lo está respecto al trabajo, a la constitución de la democracia, la paz y en general a la formación de las identidades nacionales. Caracterizar y precisar los límites de la recreación como disciplina (Mesa Cobo, 1999), supone definir, situar y contextualizar histórica, social y epistemológicamente los conceptos de ocio y tiempo libre mostrando sus relaciones y diferencias.

\section{Ludus, recreación y tiempo libre}

El ocio ha sido considerado históricamente distinto, por ejemplo, en Grecia era privilegio de la clase alta, que le veía como posibilidad de la expresión del hombre en su condición ética, pues era allí donde la creación se daba; "se le reconocen tres elementos contenidos en el ideal griego del ocio: "tiempo, necesario para la elaboración y elevación de la cultura; fermento intelectual, dado en la necesidad de explicaciones mitológicas o metafísicas constantes, y seguimiento de un ideal, premisa más característica de la Grecia antigua que de cualquier otra cultura" (Goodale y Godbey, 1988.); por tanto, agregan, el "ocio" tal como lo entendieron y usaron los primeros filósofos griegos, solamente puede ser entendido a la luz de los ideales de la cultura griega, concebido como una predisposición del ánimo, del ser, como una actitud "para" configurada en un ideal de cultura (Gerlero, 2005). El ocio hace parte del ideal griego, del cuidado de sí, de las prácticas de sí, del ocuparse del sí mismo hasta el siglo I que proclamaban, vivían y enseñaban los griegos (Foucault, 2006). También del aprender, el ocio se relacionaba con escuela, en tal sentido era de ociosos aprender (Elías y Dunning, 1992).

En Roma, en tanto, los aspectos del ocio fueron resignificados:

1-se inicia una fusión en el ámbito individual de las categorías "ocio-trabajo", reconocidas socialmente. La negación del otium romano, es el negotium, de donde deriva "negocio", es decir, trabajo al que se dedicaban negociantes y mercaderes. Ocio y negocio son parte constitutiva del hombre completo, y desde esas dos dimensiones el hombre se manifiesta; 2- el tiempo de ocio es en Roma atributo de las grandes masas -aunque no en forma exclusiva- para quienes los poderosos sirven con fiestas y espectáculos. Esto marca un perfeccionamiento del ocio popular que deriva en ocio de masas como arma de "dominación"; 3- el ocio se expresa en actividades concretas y colectivas fundamentalmente de tipo "espectáculo", no ya en términos de ideas o ideal; 4- La búsqueda del placer está impregnada de materialidad y desprovista de los parámetros de la moralidad ateniense (Elías y Dunning, 1992, p. 83).

Con la caída del Imperio romano, el cristianismo propicia la soldadura mental y moral de la sociedad con su ideal de salvación e inmortalidad de las almas. La fe reemplazará a la razón y se hará objeto de contemplación. De esta manera, la idea de ocio griega se abandona, en tanto la razón es uno de los aspectos que lo fundan. Aunado a esto, la noción del tiempo va a cambiar.

Para los cristianos el tiempo es de por sí sagrado, tiende hacia una plenitud futura, pertenece al mundo venidero, pero debido a la realidad humana deben ser signos visibles para el hombre los que marquen los tiempos sagrados; es así como se impone el domingo o "día del Señor" en el calendario litúrgico. Tanto el "descanso" como la "contemplación" y la "fiesta" representan la posibilidad material para el hombre de profundizar y tomar conciencia de su dependencia con el creador y disponerse espiritualmente a recibir su gracia. Así puede resumirse el nuevo sentido otorgado por la doctrina cristiana al tiempo de descanso, a la interrupción laboral que se impone con el domingo (Elías y Dunning, 1992, p. 94).

Si bien, como hemos visto, dar cuenta de la epistemología-historia del concepto de ocio nos envía hacia Grecia y Roma y nos sitúa en los comienzos del cristianismo -momento fundamental para entender una de tantas concepciones del mundo, del tiempo, de lo humano, de la ideología y de las condiciones sociales culturales y políticas-, permitiéndonos entender muchas cosas de nuestro presente; el periodo que más nos interesa y en el que nos vamos a centrar es la Modernidad entre los siglos XVI y XVII, pues es allí donde las instituciones sociales se configuran, produciendo por ejemplo la escuela, los discursos en torno a la invención de la infancia -que se naturalizan-, los estados nación, el progreso y luego el desarrollo, la aparición de ciencias, disciplinas y saberes, etc., situándonos finalmente en la segunda mitad del siglo XX en que dichas institucio- 
nes, saberes y poderes -aún en las regularidades- han cambiado constituyendo nuestro presente.

Durante el siglo XVI, con el incipiente surgimiento de la economía de mercado, comienza a gestarse, como derivación del ocio, el concepto de ociosidad. La condición humana adquiere la condición natural de impureza y pecado, y la potencialidad del ser humano. El trabajo pasa a ser el elemento que dignifica al hombre y la ociosidad su condena. La crítica al ocio que surge en este período abarca dos dimensiones: la económica y la moral. A partir de aquí el concepto de ocio, tal como lo hemos analizado, no tiene la capacidad de expresar un contenido válido en diferentes momentos históricos. Cuando se intenta recobrarlo, da lugar -por las condiciones históricas-, a un nuevo concepto, el tiempo libre (Elías y Dunning, 1992). Recordemos por ejemplo el lugar y la significación del tiempo en la escuela, su buen uso, en contraposición a la pereza que será fuertemente castigada. Así, la idea de tiempo libre en las sociedades preindustriales es distinta a la noción del tiempo que circuló y se instaló en la era industrial.

El surgimiento de los estados modernos sedientos de progreso, estabilidad económica y ansiosos de rendimientos óptimos, modela los apetitos de los hombres; de manera que, rezagado, abolido el tiempo sagrado, se pretende masificar el uso de todos los tiempos incluido el cotidiano, por cuanto la tradición pierde, por así decirlo, su esencia de comunión, de acercamiento familiar y social, hasta llegar a uniformar los deseos individuales y sancionar los ritmos que no sirvan a los intereses de las que se anuncian naciones modernas. Esta noción ha penetrado la escuela transformando a los sujetos que hacen parte de este espacio de clausura sumergido en una distribución meticulosa del tiempo escolar, en la proyección de los destinos y caminos elegidos para los hombres, en los roles determinados como propios de la infancia, de la adolescencia, de la adultez o de la vejez, para el hombre o para la mujer: a modo de una marca que define lo prohibido, lo indebido, lo permisible y lo justo. Una noción aprendida, una sensación que rebasa los marcos de las teorías, elemento práctico que acompaña a las rutinas y que está inserto en las actividades como testigo mudo que elocuencia y demarca los ritmos vitales. El tiempo, su buen uso, es una categoría que atraviesa la humanidad desde los ideales ilustrados y de progreso, la cual sin precisar distinciones sociales o de haberes, constituye más bien el motor que impulsa desde los más diversos afanes hasta las más densas calmas; concepto que soporta y viabiliza el diario transcurrir donde tanto el pobre como el ilustra- do, lo llevan a manera de sello que les hace pensar de una determinada forma signada por una moral de uso y rendimiento, por una gana desmedida de ahorrar los minutos al servicio del trabajo, de apurar la vida huyendo de las horas de inactividad rotulada como ocio, pereza y por ende enfermedad. Vienen en su ayuda la moral, en estrecha relación con la educación, la moral social que sobre los sujetos impondrá el disciplinamiento en el trabajo, y la crítica, así como la sanción, a la ociosidad a favor de las ocupaciones que aumenten el progreso de las naciones, contribuyendo a su industrialización (Herrera Beltrán y Buitrago, 1999).

La normalización de las actividades y las prácticas humanas será lo que rija las dinámicas sociales, las relaciones entre los sujetos, los modales, las formas de hablar, de divertirse, hasta el punto de minar la alegría y el humor; "se predicó y se legisló contra las diversiones de los pobres" (Thompson E, 1984, p. 449), contra la risa, en un intento de suprimir bailes y ferias tradicionales, como parte de la desvalorización de la comodidad, del placer y de las cosas de este mundo. Instaurado un tiempo laboral prolongado para adultos y niños, no se hicieron esperar las demandas por el tiempo libre asumidas por organizaciones gremiales de obreros. Por ejemplo, la obligatoriedad de asistir a la escuela, para el caso de los niños trabajadores, representó un tiempo de descanso de las extenuantes jornadas laborales (Varela y Álvarez-Uría, 1997).

La lucha por la conquista de tiempo libre se sucede logrando paulatinamente una reducción de las horas de trabajo. Al mismo tiempo, se diversifican las consignas para la utilización del tiempo liberado. Aparece la demanda por la necesidad de ese tiempo para el desarrollo cultural y la socialización. Se resumen aquí aspectos vinculados a la participación social, a la educación e instrucción en sociedades cada vez más complejas hasta lograr las vacaciones pagadas, reivindicación que impulsa sostenidamente el desarrollo del turismo como práctica en el tiempo libre (Gerlero, 2005).

El tiempo libre es entonces un concepto estrechamente producido desde el trabajo y como oposición a este, es decir, aquel que trabaja debe descansar y en ese tiempo, tiempo libre, se recreará, ya no como quiera; pues habrá otros sujetos especializados que sabrán más sobre sus necesidades y cómo alcanzarlas, de allí que se le otorgue el poder a dichos sujetos para planear el tiempo libre. Las actividades recreativas serán ofrecidas en abundancia, desde la empresa y la caja de compensación a la que está adscrito, la universidad en la que estudia, el barrio en el que vive; igual, proliferaran 
los sitios que venden los objetos de consumo para recrearse: juguetes, juegos, ropa e implementos deportivos, objetos muy diversos; simultáneamente aumentarán los profesionales en recreación dispuestos a asesorarlo en el uso de su tiempo, de su dinero y siempre en beneficio propio. La recreación se volverá un derecho, un servicio y un bien de consumo.

Este aumento del tiempo libre en las sociedades capitalistas responde como se observa, a una necesidad estructural del sistema: descansar para seguir produciendo y disponer de tiempo para el consumo (Gerlero, 2005). Un descanso que debe ser, a todas luces, reparador, que sirva de acondicionamiento físico para el trabajo, que represente bienestar, salud y beneficios.

Habíamos dicho que la teoría del juego presentada por Callois, en cuyo extremo del que nos ocuparemos está el ludus, campo en el que tiene lugar la recreación como la conocemos hoy, ha visto aparecer y naturalizarse un corpus teórico y un corpus práctico amplísimo, especialmente en la segunda mitad del siglo XX en una sociedad neoliberal. Desde muchos saberes y poderes, se muestran sus beneficios y se le implementa bajo la justificación de una necesidad. Así, la recreación se vive en la colectividad, de manera masiva impulsada desde los medios de comunicación, la publicidad y desde instituciones y políticas multinacionales que siguen muchos de los principios que fundamentan el juego. La recreación tendrá como propósitos la organización y el control social mediante actividades que dan salida a las pulsiones de manera regulada y beneficiosa, principalmente en el tiempo libre.

Señala Gerlero cómo "el universo recreativo existe, con su universo simbólico compartido por el conjunto de la sociedad al que los sujetos adhieren desde la convicción de que en esa adhesión particular accederán al placer buscado" (2005). A partir de dicha afirmación es posible entender la recreación -coherente con lo que hemos mostrado- como el:

Conjunto de prácticas de índole social, realizadas colectiva o individualmente en el tiempo libre de trabajo, enmarcadas en un tiempo y en un espacio determinados, que otorgan un disfrute transitorio sustentado en el valor social otorgado y reconocido a alguno de sus componentes (psicológico, simbólico, material) al que adhieren como satisfactor del placer buscado los miembros de una sociedad concreta (Gerlero, 2005 s.p.).

Así, las características esenciales del juego toman en la recreación diferentes formas de expresión, justamente por ser esta un producto histórico social. Se integra así la vertiente histórica que vincula a la recreación con necesidades y habilidades presentes en el mundo laboral y las propias del juego, expresión de libertad por excelencia. En este sentido, las ludotecas van a ocupar un lugar importante en lo que se refiere a la recreación, primero de la infancia, con algunos matices particulares nacen para la infancia necesitada y desprotegida, para los grupos humanos desplazados y en riesgo, pasando a constituirse en actividades que comparten fines con las bibliotecas e instituciones educativas como las universidades, pero también con actividades de los barrios, las asociaciones, las organizaciones sociales, las cajas de compensación familiar, ocupadas del bienestar, la salud de la familia, la infancia, la juventud y el adulto mayor (Gerlero, 2005).

Una revisión a la política de investigación de Colciencias en Colombia (Plata Cabiedes, 1999) muestra al deporte y la recreación como ciencias sociales y humanas, incluidas en el programa nacional de ciencias sociales y humanas que resultó de la evaluación desarrollada entre 1991 y 1998. En relación con la emergencia de la lúdica el deporte y la recreación:

Se han precisado unos principios orientadores de la labor del fomento en el ámbito del programa; estos emergen y se construyen a partir de la reflexión iniciada en el Recinto Quirama, en 1992, que se publicó bajo el sugerente título de Los retos de la diversidad Colciencias, Tercer Mundo, 1993. Estos principios son: a. Reconocer la diversidad teórica y metodológica de las disciplinas sociales y humanas. b. Destacar, como factor de desarrollo de las ciencias sociales y humanas, la consolidación de una comunidad de investigadores con autonomía e identidad. c. Valorar el papel que juega el Estado en la promoción de la investigación en aquellas modalidades no demandadas por el mercado. d. Reconocer la contribución de estas ciencias en la formación de una sociedad más justa y equitativa. e. Propender por desconcertar la investigación e impulsar las regiones.

Existen algunos proyectos de investigación en curso relacionados con el juego, la recreación, el deporte y el uso del tiempo libre..., lo primero que se debe indicar es su carácter multi-e interdisciplinario.

En este documento se señala que el campo del juego y la recreación tiene relaciones importantes con los estudios cognitivos, entre el Centro de Investigaciones en Psicología, Cognición y Cultura, bajo la dirección de la doctora Rebeca Puche y en una perspectiva piagetiana, con el apoyo de la Universidad del Valle y de Colciencias (Plata Cabiedes, 1999). 
Otras definiciones de la recreación son las que la precisan como actividad que renueva al individuo del trabajo o como un programa. Para Pablo Waichman, la recreación es:

La diversión recreadora, autocondicionada, funcional, libre para, es la plena y consciente entrega a algo por sí mismo y no por necesidad, lo que autoafirma en diversos aspectos a la persona en cuanto sujeto recreador de sí mismo y de lo que lo rodea (2000, p. 104).

Concepción basada en la idea de tiempo libre como libertad: "el tiempo libre consiste en un modo de darse tiempo social. Personalmente sentido como libre y por el que el hombre se autocondiciona para compensarse, y en último término afirmarse individual y socialmente" (2000, p. 104). Señala a otros autores y algunas definiciones que amplían algo más el espectro de la que viene construyendo, por ejemplo, al reconocer la recreación como cualquier actividad también del tiempo de ocio no solo del tiempo libre. De otro lado, la consideración de una actividad socialmente aceptada entra en contradicción con las actividades del tiempo del ocio, ya que ellas responden a los intereses especialmente individuales, por lo que no necesitan de la aprobación social para ser vividos (Waichman, 2000). En otro sentido, que sea una actividad elegida libremente, por cuanto puede significar la obtención de valores sociales y no solo personales, riñe con la idea de actividad en el tiempo de ocio, ya que este se le considera un tiempo en el que es posible alcanzar satisfacciones personales inmediatas a mediano o largo plazo, pero fundamentalmente de orden personal. En tal sentido, aclara Waichman (2000), el ocio es un periodo de tiempo, la recreación es un contenido de este tiempo (en actividad), aun cuando están íntimamente relacionados no son sinónimos.

Podríamos concluir tempranamente que la recreación emerge en el seno de la sociedad capitalista como la actividad en el tiempo libre opuesto al trabajo,constituyéndose en una categoría política que regula las prácticas sociales actuando como una economía de la vida.

Se asiste al ofrecimiento cada vez más sofisticado e invasivo de programas de: recreación pública, recreación privada, recreación comercial, urbana o rural. Entre los contenidos de los programas de recreación ${ }^{22}$ aparece la expresión físico deportiva: deportes, y actividades de acondicionamiento físico, expresión al aire libre, expresión acuática, expresión lúdica: todas las formas de juego, incluyendo proyectos elaborados, como son las ludotecas. También, como expresión manual, expresión artística, expresión conmemorativa, expresión social, expresión literaria, expresión de entretenimientos y aficiones, expresión técnica: actividades de enriquecimiento personal y ajuste a la vida, habilidades de autodefensa, reparación de cosas en casa, expresión comunitaria, etc.

Los sujetos a los que van dirigidos estos programas recreativos son los infantes: niños y niñas, adolescentes, jóvenes, adultos mayores, discapacitados, etc., algunas representan una inversión económica, otras no. Los espacios son abiertos: patios, calles, plazas, centros deportivos, áreas verdes, zonas de bosques, las cuales son frecuentadas y utilizadas por las clases marginadas, y cerrados, como son los cines, boliches, centros deporti-

22 Se revisaron, políticas, leyes, programas, lineamientos, proyectos, experiencias entre las cuales podemos citar: Mesa Cobo, Guillermina: La recreación: algo más que volver a hacer. Universidad del valle. I Simposio de Investigación y Formación en Recreación. Septiembre 30 a Octubre 2 de 1999. Pereira, Colombia. Vicepresidencia de la República. Coldeportes. http://www.redcreacion.org/documentos/simposio1if/GMesa.html/ 2 de 1999. Pereira, Colombia. Vicepresidencia de la República. Coldeportes. FUNLIBRE. http://www. redcreacion.org/documentos/simposio1if/GMesa.html/

Educación para Todos: cumplir nuestros compromisos comunes. Texto aprobado por el Foro Mundial sobre la Educación. Dakar, Senegal, 26-28 de abril de 2000. http://www.unesco.org/education/efa/ ed_for_all/dakfram_spa.shtml Fundación Corona: Cuarenta años de la Fundación.

Gestión social con imaginación. 2003.http://www.fundacioncorona. org.co/descargas/historia_FC.pdf Indeportes Antioquia. INDER http:// www.indeportesantioquia.gov.co/index.php?option=com_content\&vie w=article\&id=355\&ltemid=141 Las niñas y los niños de Medellín ya tienen megaludoteca 27-12-2007 http://www.medellin.gov.co/alcaldia/jsp/modulos/boletines/RBoletin.jsp?IDBOLETIN=2038-2007

LEY 181 DE 1995 LEY DEL DEPORTE: "Por la cual se dictan disposiciones para el fomento del deporte, la recreación, el aprovechamiento del tiempo libre y la educación física y se crea el sistema nacional del deporte". (Nota: Modificada en lo pertinente por la Ley 344 de 1996 artículo 44).http://www.redcreacion.org/documentos/ley181.htm Lineamientos Técnicos y Operativos de las Ludotecas Naves. Documento elaborado por FUNLIBRE para el ICBF y la corporación día del niño. capítulo 2. Componente técnico. Parte 1 de 2 del Capítulohttp:// www.redcreacion.org/documentos/ludotecas/Comptec1.html

Ludotecas Bogotá Distrito Capital. P. 1. http://www.alcaldiabogota. gov.co/sisjur/normas/Norma1.jsp?i=21394

Política pública nacional de primera infancia "COLOMBIA POR LA PRIMERA INFANCIA" Ministerio de la Protección Social. Ministerio de Educación Nacional. Instituto Colombiano de Bienestar Familiar DNP-DDS-SS Versión aprobada Bogotá, DC., 03 de diciembre de 2007 DOCUMENTO COMPES SOCIAL 109. Consejo Nacional de Política Económica Social. República de Colombia. Departamento Nacional de Planeación. www.dnp.gov.co/PortalWeb/Portals/0/archivos/.../109.pdf- 
vos y culturales, casas de fin de semana, etc. El personal que imparte o dirige la recreación viene de diversas profesiones sociales con estudios en el campo concreto de desempeño, entre ellos -pioneros de ésta especialidadlos profesionales de la educación física, con formación técnica y tecnológica pues la formación de posgrado no se ha hecho presente todavía en el país, como si lo ha hecho en México, por ejemplo (Aguilar Cortés, 2000).

En la década de los sesenta, además de los discursos a favor de la recreación venidos de instituciones como las cajas de compensación familiar creadas en la década de los cincuenta simultáneamente a la implementación del sistema de subsidio familiar y que tenían a cargo el bienestar de los empleados ofreciendo desde 1962 actividades de bienestar social, también se expresó a favor de estas actividades el Ministerio de Trabajo y Bienestar Social ${ }^{23}$.

En la actualidad, la recreación ha entrado en el grupo de las disciplinas humanas con un cuerpo teórico antropológico y social en construcción que se desliza por fuera también de la institución escolar y que en el contexto de la educación física cumple cada vez más unos fines socializadores más etnoeducativos y ambientales y que como derechos aparecen en las agendas sociales que no acaban de cumplirse. De allí su puesta en acción desde diversos planes de los llamados e institucionalizados países del tercer mundo.

\section{Corolario}

El juego, como una práctica connatural al ser humano donde el rito tiene lugar, ha sido regulado desde la educación en el sentido de usarlo para alcanzar fines propuestos desde diversos saberes. Esa normalización y administración del tiempo libre de los sujetos en aras de una mayor y mejor producción ha situado al juego

23 En 1957, la Andi convocó a sus afiliados en Bogotá y creó la Caja Colombiana de Subsidio Familiar, Colsubsidio. En el resto del país se constituyeron simultáneamente otras cajas de compensación familiar auspiciadas por diferentes gremios: comerciantes, banqueros, aseguradores, etc. La función básica de las Cajas en sus inicios, se limitaba, según lo ordenaban las normas vigentes en ese entonces, a recaudar los aportes patronales para repartirlos mensualmente en cheques, a los trabajadores de las empresas afiliadas. En el año 1962 el Ministerio de Trabajo y Seguridad Social, a través del decreto 3151 del mismo año, abrió las puertas a los servicios sociales de las Cajas de Compensación Familiar; fue así como nacieron los servicios de salud, mercadeo, educación, vivienda, recreación y crédito de fomento para industrias familiares. Fue expedida la Ley 21 de 1982, donde se ratifica el carácter de prestación social que tiene el subsidio familiar y se amplió la cobertura de este régimen, extendiéndose a toda la población asalariada del país, se reiteró el carácter de corporaciones a las cajas de compensación, se les asignaron funciones de seguridad social. Recuperado de http://www.colsubsidio.com/es_caja/index.html\#d en el lugar de la práctica recreativa por excelencia, es decir, desde el lado del ludus -entendido como juego dirigido de orden social por excelencia-, antes que del lado de la paideia -comprendido como juego libre, que desde entonces permanecerá invisibilizado y constreñido a la práctica individual e íntima-. El juego libre es cada vez menos posible, en tanto la regulación mediante dispositivos que aseguran el buen uso del tiempo libre a partir de actividades aceptadas socialmente se hace mayor; actividades sobre todo dirigidas por otros, bajo la denominación de recreación en detrimento del juego para el tiempo de ocio, que ha ido desapareciendo de la vida de las personas. El juego ha sido regulado y puesto a funcionar dentro y fuera de la escuela como estrategia didáctica y en el tiempo libre, fuera del marco de la escolarización, bajo la denominación de ludus. Esas actividades lúdicas serán cada vez más homogéneas en detrimento de la propia individualidad, cuya elección constituirá más el deseo de los otros, olvidando el suyo propio o desconociendo que ha tenido en algún momento un interés que le hace distinto frente al grupo.

\section{Referencias}

Aguilar Cortés, L. (2000). FUNLIBRE. Recuperado de http://www.redcreacion.org/relareti/documentos/ perfiles_profesionales.html

Aries, P. (1987). El niño y la vida familiar en el Antiguo Régimen. Madrid: Taurus.

Barreau, J. J. (1991). Epistemología y antropología del deporte. Madrid: Alianza Editorial.

Caillois, R. (1986). Los juegos y los hombres (la máscara y el vértigo). México: Fondo de Cultura Económica.

Congreso de la República de Colombia. (2004). Acto Legislativo 2. (D. 0. 45.775., Ed.) Recuperado de . http:// www.secretariasenado.gov.co/senado/basedoc/cp/ acto_legislativo_02_2004.html

Consejo Iberoamericano del Deporte. (1994). presidencia gov.co. Recuperado de http://web.presidencia.gov.co/ leyes/2007/septiembre/ley1160260907.pdf

Deleuze, G. (1989). Michel Foucault-Filósofo: ¿Qué es un dispositivo? Ed. Du Seil.

Del Real y Mijares, M. (1906). Escuela de niñas. Revista de la Instrucción Pública, 7-8(XIX), 90.

Duvignaud, J. (1982). El juego del juego. México: Fondo de Cultura Económica.

Elías, N., y Dunning, E. (1992). Deporte y ocio en el proceso de civilización. Madrid: Fondo de Cultura Económica.

Escobar, A. (2007). La invención del tercer mundo. Construcción y deconstrucción del desarrollo. Caracas: Fundación Editorial El Perro y la Rana.

Foucault, M. (1990). Tecnologías del Yo y otros textos afines. Barcelona: Paidós. 
Foucault, M. (2006). Hermeneútica del sujeto. Bogotá: Fondo de Cultura Económica.

Gerlero, J. (Octubre de 2005). Diferencias entre ocio, tiempo libre y recreación: lineamientos preliminares para el estudio de la recreación. Recuperado de http:// www.redcreacion.org/documentos/cmeta1/JGerlero. html/

Goodale, G. (1988.). The Evolution of Leisure. State College. PA, EEUU: Venture Publishing.

Herrera Beltrán, C. y Buitrago, B. (1999). El cuerpo del niño al interior de la organización temporal de la escuela primaria en Colombia entre 1870 y 1890. Revista Educación y Pedagogía, 23-24.

Herrera Beltrán, C., y Buitrago, B. (2011). El juego y la escuela en Colombia a finales del siglo XIX. Revista Pedagogía y Saberes, 33, 63-71.

Martínez Boom, A., Noguera Ramirez, C., y Castro, J. (2003). Currículo y modernización. Cuatro décadas de modernización en Colombia. Bogotá: Magisterio.

Mesa Cobo, G. (1999). Funlibre. Recuperado de http:// www.redcreacion.org/documentos/simposio1if/ GMesa.html

Ministerio de Educación Nacional. (1968). Decreto Número 2743. Recuperado de http://www.mineducacion. gov.co/1621/articles-104216_archivo_pdf.pdf

Plata Cabiedes, J. (1999). Juego y conocimiento. Apuntes para una agenda investigativa desde las ciencias sociales y humanas. Programa Nacional Ciencias Sociales y Humanas / Colciencias. Recuperado de http://www. redcreacion.org/documentos/simposio1if/JJPlata.html
Sáenz, J. (1997). Mirar la infancia: Pedagogía moral y modernidad en Colombia 1903-1946. Medellín: Ediciones Foro Nacional por Colombia.

Saldarriaga, O. (2003). Del oficio del maestro. Bogotá: Magisterio.

Serrano Sánchez, J. (1992). Revista Apunts. Educació física i esports, 29. Recuperado de http://articulos-apunts. edittec.com/29/es/029_018-030_es.pdf

Thompson E. (1984). Tradición, revuelta y consciencia de clase. Estudios sobre la crisis de la sociedad preindustrial. Barcelona: Grijalbo.

UNESCO. (1976). Conferencia General de las Naciones Unidas para la Educación, la Ciencia y la Cultura. Recuperado de http://unesdoc.unesco.org/ images/0011/001140/114038S.pdf

UNESCO. (1978a). Conferencia General de las Naciones Unidas para la Educación, la Ciencia y la Cultura. Recuperado de http://articulos-apunts.edittec.com/29/ es/029_018-030_es.pdf

UNESCO. (1978b). UNESCO. Recuperado de http://portal. unesco.org/es/ev.php-URL_ID $=13150 \& U R L \_D O=D O$ _ TOPIC\&URL_SECTION=201.html

Varela, J., y Álvarez-Uría, F. (1997). Arqueología de la escuela. Madrid: La Piqueta.

Waichman, P. (2000). Tiempo libre y recreación. Revista Kinesis, 104. 